\title{
Les cancers orphelins et nos obligations morales
}

$\mathrm{P}$ renons un garçon de 6 ans atteint d'un neuroblastome avec métastases et qui a besoin d'une chimiothérapie expérimentale de phase I, une femme de 23 ans chez qui il a fallu 6 mois pour diagnostiquer un sarcome non différencié à la suite de multiples consultations de médecins, ou un homme de 45 ans atteint d'un cancer du sein et qui n'a pas de groupe d'entraide de pairs. Chacune de ces personnes est mal servie par le système de santé du Canada. Ces cas illustrent le défi que nous devons relever comme société pour définir nos valeurs et les priorités de notre justice distributive lorsque vient le temps d'attribuer les crédits à consacrer à la recherche et aux soins de santé dans un contexte de budgets fixes.

On définit en général les maladies rares - plus de 6000 - par un critère : elles atteignent moins d'une personne pour 2000 habitants ${ }^{1}$. Tous les cancers pédiatriques et beaucoup de cancers rares chez l'adulte correspondent à cette définition. On reconnaît plus précisément que les adolescents et les jeunes adultes (et en particulier les jeunes femmes) atteints de cancer constituent une population orpheline, peu représentée dans les essais cliniques, chez qui les résultats sont sous-optimaux et dont les cancers sont insuffisamment étudiés sur le plan biologique.

On croit que l'incidence négative sur la survie et la qualité de vie que connaissent les personnes atteintes d'un cancer rare peut être attribuable au diagnostic tardif, au manque d'accès à des soins spécialisés, au manque de groupes d'entraide ou à des choix de traitement inadéquats. La façon de trancher le dilemme posé par l'utilisation responsable de ressources limitées pose un défi énorme sur le plan de l'éthique ${ }^{2}$. Nous devons prendre position publiquement et définir des procédures équitables et transparentes permettant aux décideurs d'établir un équilibre entre ce que nous devons comme société aux enfants par rapport aux personnes âgées, de gérer des ressources limitées dans la prise de décisions en fin de vie, de pondérer les preuves des avantages et de communiquer ces décisions difficiles avec compassion. Nous devons relever le défi qui consiste à recueillir ces points de vue auprès de tous les groupes intéressés et de la résumer de façon équitable.

Le traitement du cancer a connu récemment des succès, mais les succès sont moins nombreux dans le cas des maladies plus rares. La mise au point de médicaments orphelins a souvent du retard sur celle d'agents plus commercialement viables. Et même lorsque l'on crée un produit, son coût peut être prohibitif. La législation sur les médicaments orphelins aux États-Unis, en Australie, au Japon et dans l'Union européenne prévoit toute une brochette d'incitations pour stimuler l'intérêt des sociétés pharmaceutiques. Il est clair que ces lois favorisent la création de médicaments et qu'elles ont un effet sur la mortalité attribuable aux maladies rares.

On a critiqué l'absence d'une politique unifiée enchâssée dans la loi au Canada et il est à espérer que le Partenariat canadien contre le cancer annoncé récemment, dont les objectifs consistent à réduire le nombre de nouveaux cas de cancers, à améliorer la qualité de vie et à réduire le taux de mortalité chez les personnes atteintes de cancer, fera progresser le programme général de lutte contre le cancer tout en corrigeant certaines des iniquités. Parallèlement, le rapport provisoire sur la Stratégie nationale relative aux produits pharmaceutiques met en évidence 5 priorités clés : la protection contre le coût catastrophique des médicaments, les médicaments coûteux contre des maladies rares, un formulaire national commun, des stratégies d'établissement des prix et d'achat, et la sécurité des médicaments ${ }^{3}$. Ce document présente des buts et des recommandations louables, y compris un cadre de gestion des médicaments coûteux contre des maladies rares regroupant des données probantes, des facteurs éthiques ${ }^{4}$ et la nécessité d'harmoniser les systèmes de réglementation et de remboursement. Il faut procéder de toute urgence à leur explication et leur adoption dans la pratique.

Même si la mise au point de médicaments orphelins domine parfois la discussion, les patients atteints de cancers rares sont victimes d'autres injustices. Comment stimuler l'intérêt pour des maladies que l'on ne rencontre qu'une fois tous les 5 ans? Il faut consacrer des ressources à une amélioration de la capacité de reconnaître les cancers rares, à regrouper des centres d'excellence, à raccourcir les temps de diagnostic et à maximiser les ressources axées sur les patients. Les organismes subventionnaires (à la fois du secteur public, comme les Instituts de recherche en santé du Canada, et du secteur privé) doivent s'attaquer spécifiquement aux iniquités en recherche.

Nous devons veiller à renforcer les soins à domicile et les soins communautaires - il ne sert à rien d'accorder toute l'importance aux soins tertiaires si l'on ne dispose pas de soins communautaires et à domicile dotés de ressources appropriées. Même si les défis que posent la prestation de soins à domicile adéquats ne sont pas toujours particuliers aux cancers orphelins, on est plus susceptible de dégager des possibilités dans les soins prévus au programme des maladies plus courantes. La Loi canadienne sur la santé appuie le concept selon lequel les patients qui ont besoin de soins spécialisés ne doivent pas avoir à supporter de fardeaux financiers disproportionnés uniquement parce qu'ils se trouvent à vivre à un endroit peu commode.

Avec la contribution vitale de la population, les décideurs du Canada doivent confronter les frontières morales difficiles de nos obligations envers ceux et celles qui sont atteints de cancers ou autres maladies rares. Ces décisions doivent se prendre dans le contexte des responsabilités des médecins, de la viabilité économique, de l'équité nationale, de la juste répartition des possibilités et de nos obligations mondiales, mais il faut concentrer clairement notre attention sur la personne.

\section{Conrad V. Fernandez}

Département de pédiatrie et de bioéthique Centre de santé IWK et Université Dalhousie Halifax (N.-É.)

Cet article a fait l'objet d'un examen par les pairs.

\section{RÉFÉRENCES}

I. van Weely S, Leufkens H. Orphan diseases. Dans : Priority Medicines for Europe and the World Project: "A public health approach to innovation." Département de la Politique et des normes pharmaceutiques (DNP). Organisation mondiale de la santé. 2005. Disponible : mednet3.who.int/prioritymeds/report/background/rare _diseases.doc (consulté le ig décembre 2006).

2. Gericke CA, Riesberg A, Busse R. Ethical issues in funding orphan drug research and development. JMed Ethics 2005;31:164-8.

3. Groupe de travail ministériel fédéral/provincial/territorial sur la Stratégie nationale relative aux produits pharmaceutiques. Rapport d'étape sur la Stratégie nationale relative aux produits pharmaceutiques. Ottawa : Sa majesté du chef du Canada; 2006. Disponible : www.hc-sc.gc.ca/hcs-sss/alt_formats/hpb-dgps/pdf/pubs/2006 -nps-snpp/2006-nps-snpp_f.pdf (consulté le ig décembre 2006).

4. MacPherson C, Kenny NP. Ethical issues in priority setting. Produit pour le Groupe de travail de la Stratégie nationale relative aux produits pharmaceutiques sur les médicaments onéreux pour les maladies rares, août 2005. 